\title{
Attentional bias modification in social anxiety: Effects on the N2pc component
}

\author{
Mario Reutter ${ }^{\mathrm{a}, *}$, Johannes Hewig ${ }^{\mathrm{a}}$, Matthias J. Wieser ${ }^{\mathrm{b}}$, Roman Osinsky, ${ }^{\mathrm{c}, *}$ \\ ${ }^{\text {a }}$ University of Würzburg, Germany \\ ${ }^{\mathrm{b}}$ Erasmus University Rotterdam, Netherlands \\ ${ }^{\mathrm{c}}$ University of Osnabrück, Germany
}

\section{A R T I C L E I N F O}

\section{Keywords:}

Attentional bias modification

N2pc

SPCN

Social anxiety

Dot probe

Reaction times

Reliability

\begin{abstract}
A B S T R A C T
Several meta-analyses to date have confirmed the efficacy of attentional bias modification (ABM) in shifting reaction times away from threatening stimuli, reducing anxiety symptoms, and buffering against stressor vulnerability. The reliability of reaction time differences, however, has been found to show unacceptable psychometric properties. In this study, we tested the impact of an extensive Dot-Probe ABM procedure, consisting of close to 7000 trials, concurrently with behavioral and electrophysiological measures within a large sample of over 100 highly socially anxious participants. Results indicated that the N2pc component demonstrates superior internal consistency and more statistical power in detecting attentional biases and their modification than reaction time (RT) differences. RTs were neither indicative of an attentional bias before ABM nor of a modification over time. In contrast, the N2pc indexed both an initial attentional preference for threatening stimuli and an alteration of this relationship after training. Outcomes were not specific for attentional training away from threat but also occurred in the no-contingency control procedure, casting doubt on the theoretic underpinnings of ABM. Electrophysiological measures are an important complement to the ABM literature and should be further utilized to assess attentional biases with excellent reliability.
\end{abstract}

With a lifetime prevalence of almost $30 \%$, anxiety disorders are the most common class of psychopathology in the United States (Kessler et al., 2005). In Europe, 100 million people (i.e., $14 \%$ of the population) are affected by anxiety disorders each year (Wittchen et al., 2011). Extensive research on clinical and subclinical samples has established a connection between anxiety and biases in the processing of fear-relevant material (Bar-Haim, Lamy, Pergamin, Bakermans-Kranenburg, \& van IJzendoorn, 2007; Koster, Crombez, Verschuere, \& Houwer, 2004). Investigations of a causal link between anxiety symptomatology and biased perception already started over 15 years ago (Mathews \& MacLeod, 2002). Since then, attentional bias modification (ABM) has been proposed as an efficient enhancing tool for anxiety treatment strategies (Bar-Haim, 2010) and also as a means of prevention due to its potential reduction of anxiety vulnerability (See, MacLeod, \& Bridle, 2009).

Several meta-analyses to date have confirmed the efficacy of ABM in shifting attentional biases, reducing anxiety symptoms, and buffering against stressor vulnerability (Jones \& Sharpe, 2017). However, effects are only small to moderate and symptom reduction seems to be better achieved by modification of interpretative instead of attentional biases.
Optimally, ABM is performed on young adults, who are trained in the lab and assessed by a clinician (Jones \& Sharpe, 2017; Price et al., 2016). While ABM protocols usually aim to train attention away from threat, recent work has suggested that in a threatening context, e.g. combat deployment of soldiers, ABM toward threat can protect against stress symptoms due to more adaptive threat-monitoring (Shechner \& Bar-Haim, 2016).

The most prevalent protocol to assess and modify attentional biases is based on the Dot Probe Paradigm (MacLeod, Mathews, \& Tata, 1986). Depending on the employed variant, subjects indicate the location or type of a dot stimulus as quickly and accurately as possible. Prior to target presentation, however, two distracting stimuli are shown - one being emotionally relevant and the other comprising a control condition. The attentional bias is then quantified as the difference between the mean response times toward dots co-located with a) the emotional distractors and b) the control category.

The psychometric properties of reaction time differences (henceforth "RT bias") have rarely been investigated. Those researchers who have quantified reliability scores of RT bias, however, found unacceptably low values (Dear, Sharpe, Nicholas, \& Refshauge, 2011;

\footnotetext{
${ }^{*}$ Corresponding author. Department of Psychology I, Julius-Maximilians-University, Marcusstr. 9-11, 97070, Würzburg, Germany.

E-mail address: mario.reutter@uni-wuerzburg.de (M. Reutter).
} 
Price, Greven, Siegle, Koster, \& Raedt, 2015; Puls \& Rothermund, 2017; Reutter, Hewig, Wieser, \& Osinsky, 2017; Rodebaugh et al., 2016; Schmukle, 2005; Staugaard, 2009; Van Bockstaele et al., 2011; Waechter, Nelson, Wright, Hyatt, \& Oakman, 2014; Waechter \& Stolz, 2015), rendering RT bias unsuitable for research on individual differences and thus for differential indication. Possibly due to this unreliability, symptom changes hardly correlate with alterations in RT bias (Jones \& Sharpe, 2017). According to the very core idea that modification of attentional biases induce these symptom changes, however, such an association should be present. If the problem does not lie within the validity of the theoretic construct, other operationalizations of attentional bias might be better suited to reveal the connection between biased processing and anxiety. For example, eye-movements have shown tremendously better reliability than reaction time differences in a dot probe task (Waechter et al., 2014) but also showed little association with anxiety symptoms. Neural measures usually dominate reaction time differences with respect to their reliability as well (Kappenman, Farrens, Luck, \& Proudfit, 2014; Kappenman, MacNamara, \& Proudfit, 2015; Reutter et al., 2017; White et al., 2016). While they are not correlated with scores of general trait anxiety (Kappenman et al., 2014), they do show an association with measures of specific anxiety (Reutter et al., 2017).

Among assessments of brain activity, the N2pc component in the EEG is a promising signal for applications within a Dot Probe Paradigm due to its potential to reveal deployment of spatial attention (Eimer, 1996; Luck \& Hillyard, 1994). The N2pc also occurs automatically and is therefore well suited to index attentional affordance of task-irrelevant stimuli (Eimer \& Kiss, 2007; Fenker et al., 2010). More specifically, it is supposed to reflect selective visual processing but not attentional shifts (Kiss, van Velzen, \& Eimer, 2008). Using facial stimuli, the N2pc is more pronounced when attending to angry compared to happy (FeldmannWüstefeld, Schmidt-Daffy, \& Schubö, 2011) or neutral (Weymar, Löw, Ohman, \& Hamm, 2011) expressions, especially if subjects are highly anxious (Fox, Derakshan, \& Shoker, 2008; Wieser, Hambach, \& Weymar, 2018). Recently, the N2pc has been successfully utilized as an index of attentional bias within a Dot Probe Paradigm, demonstrating higher statistical power and greater reliability than reaction time differences (Kappenman et al., 2014, 2015; Reutter et al., 2017). It has also been employed to assess the impact of a one-session attentional bias modification training (Osinsky, Wilisz, Kim, Karl, \& Hewig, 2014).

In the current study, we aimed to investigate the efficacy of an extensive attentional bias modification procedure concurrently via behavioral and electrophysiological measures (i.e., reaction time differences and the N2pc, respectively) in a highly socially anxious but undiagnosed sample. We hypothesized that subjects in the training condition show greater reductions of attentional bias than control participants and that these changes are more pronounced in the N2pc than in reaction times. The cross-sectional data prior to training including a correlational analysis of attentional bias measures and questionnaire data have been reported elsewhere (Reutter et al., 2017).

\section{Method}

\subsection{Participants}

One hundred twenty-eight subjects (99 female; mean age $M=23.0$, $S D=3.2)$ started participation in the experiment for $10 €$ per hour (totaling to $150 €$ if the study was finished). Participants were selected after completion of an online screening questionnaire for elevated but non-clinical social anxiety consisting of five items on a 5-point scale based on the DSM-IV criteria for social phobia (cp. Ahrens, Mühlberger, Pauli, \& Wieser, 2015; Reutter et al., 2017; Wieser \& Moscovitch, 2015). Subjects with a mean item score of 3.2 or higher (i.e., the upper $19.32 \%$ of all 1152 individuals screened) were invited to participate in the main experiment. Of the 128 individuals who accepted our invitation and started the main experiment, 3 participants reported to have a diagnosed mental disorder not until completion of the first experimental session, 9 dropped out during the training or didn't meet the required frequency of training sessions (see Training Conditions), for 9 individuals too few artifact free trials per condition could be obtained during any EEG recording, and with 6 participants technical or other issues occurred during one of the EEG measurements. After these exclusions, 101 participants were left for our final analysis. All participants of the final sample reported to be free of any diagnosed mental or neurological disorder and gave written informed consent. The study was approved by the local ethics committee of the Würzburg Department of Psychology and was in accordance with the Declaration of Helsinki.

\subsection{Stimulus materials and procedure}

Attentional biases were assessed at three time points: pre, post, and eleven weeks following up an attentional bias modification training using a modified Dot Probe Paradigm (MacLeod et al., 1986). During each of these sessions, subjects completed four different questionnaires targeting general and social anxiety in paper form and a fixed order: the trait version of the State Trait Anxiety Inventory (STAI; Spielberger, 2010; German version: Laux, Glanzmann, Schaffner, \& Spielberger, 1981), the Social Interaction Anxiety Scale (SIAS; Mattick \& Clarke, 1998; German version: Stangier, Heidenreich, Berardi, Golbs, \& Hoyer, 1999), the Social Phobia and Anxiety Inventory (SPAI; Turner, Beidel, Dancu, \& Stanley, 1989; German version: Fydrich, Scheurich, \& Kasten, 1995), and the Social Phobia Scale (SPS; Mattick \& Clarke, 1998; German version: Stangier et al., 1999). Subsequently, participants also completed the Beck Depression Inventory (BDI; Beck, Steer, \& Brown, 1996) in the German short version BDI-V (Schmitt \& Maes, 2000) and the Big Five Aspects Scale (BFAS; DeYoung, Quilty, \& Peterson, 2007; German Version: Mussel \& Paelecke, in prep.).

After the questionnaires, the behavioral task was run on a desktop computer, using PsychoPy v1.81.00 (Peirce, 2007) as presentation software. The computer task comprised a standard Dot Probe Paradigm (MacLeod et al., 1986). Trials started with a fixation cross in the center of the screen for a random duration of 750-1250 ms (rectangular distribution with 42 steps due to monitor refresh rate of $85 \mathrm{~Hz}$ ). In succession, angry and neutral faces were presented in left and right visual hemifields for $500 \mathrm{~ms}$, followed by a target co-located with one of the two faces. All stimuli were centered at $8.1^{\circ}$ visual angle from the fixation cross with the faces covering an area of $8.5^{\circ} \times 11.4^{\circ}$ (width $\times$ height; based on a distance of $53 \mathrm{~cm}$ from the screen). Subjects were instructed to keep their eyes on the fixation cross the whole time and indicate via button press as fast and as accurately as possible if the target consisted of an upright colon (":") or was rotated by $90^{\circ}$ (". "). Moreover, participants were told that during each trial two pictures will be shown prior to the dot targets but it was clear from the instruction that they are irrelevant for the task. They were not explicitly instructed, however, to ignore the pictures. The probe disappeared after button press or a maximum of $1000 \mathrm{~ms}$ resulting in marking the trial as incorrect. Participants completed ten training trials that were repeated as long as more than two mistakes were made. RTs were recorded for two blocks, separated by a self-paced break, with 216 trials each resulting in a total number of 432 trials per EEG session. The sequence of a single trial is illustrated in Fig. 1.

The Karolinska directed emotional faces database (KDEF; Lundqvist, Flykt, \& Öhman, 1998) was utilized for the choice of facial stimuli. We selected 18 pairs of angry and neutral facial expressions of the same individual ( 9 female) such that both emotions exhibited very good perceptibility according to Goeleven, Raedt, Leyman, and Verschuere (2008). This resulted in angry and neutral expressions being extracted from models F01, F03, F07, F09, F19, F20, F22, F26, F32, M02, M05, M06, M10, M11, M14, M24, M25, and M29. For practice trials, models F28 and M08 were used. Three pair-conditions were created per individual: angry-neutral, neutral-angry, and neutral-neutral. 


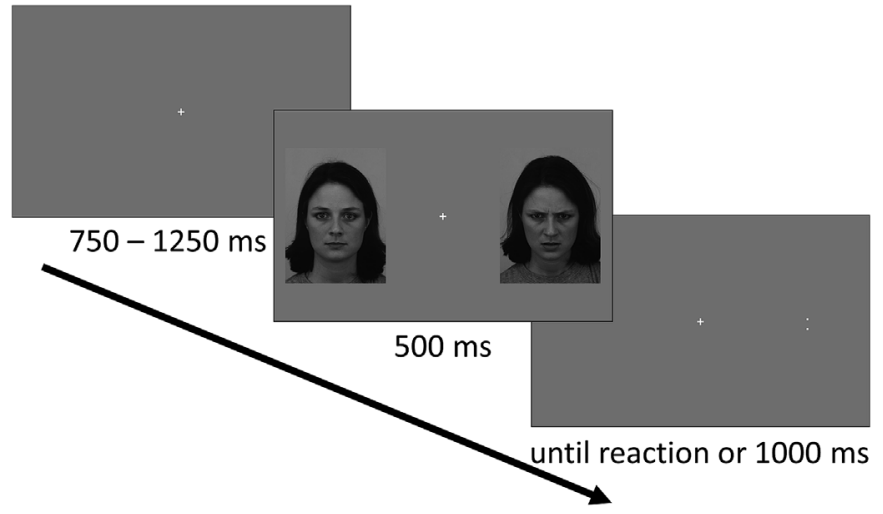

Fig. 1. Example of a single trial procedure with angry face and target on the right. Location of the facial expressions and targets as well as target kind (":" vs. “. .") were counterbalanced.

Furthermore, we balanced target location (left vs. right) and target category (":" vs. “. ") resulting in the aforementioned 18 Models $\times 3$ Distractor Conditions $\times 2$ Target Locations $\times 2$ Target Categories $=216$ trials per block. The two distractor conditions containing an angry facial expression were later used for data analysis.

Before and after the behavioral task, participants indicated their mood on a 5-point self-assessment manikin scale (SAM; Bradley \& Lang, 1994) using the dimensions valence and arousal. Subsequent to the mood rating, they rated the 36 facial stimuli they had encountered during the Dot Probe Task. Finally, demographic data were assessed.

\subsection{Training conditions}

The manipulation of our study consisted of eight sessions of ABM vs. control procedure also based on the Dot Probe Paradigm (MacLeod et al., 1986). Subjects in the ABM condition received a modified version of the Dot Probe with targets appearing only in the location of neutral facial stimuli (i.e., $100 \%$ contingency away from threat) while control participants executed a standard Dot Probe Paradigm (i.e., 50\% contingency). Each session took place on different days within a time window of two to four weeks (i.e., at least two appointments per week but a maximum of four). During every training day, four blocks with 216 trials each were administered, totaling to 864 trials per session and 6912 trials per participant. The trial structure during the training was identical to the one during the EEG recordings except for a $1 \mathrm{~s}$ feedback after erroneous or missing responses during training in order to increase motivation.

\subsection{EEG recording and processing}

The electroencephalogram (EEG) was recorded during three time points before, after, and 11 weeks following up the training procedure with a sampling rate of $500 \mathrm{~Hz}$ and a bandpass filter of $0.1-250 \mathrm{~Hz}$ at 31 scalp sites (Fp1, Fp2, F3, F4, F7, F8, F9, F10, Fz, C3, C4, CP1, CP2, P3, P4, P7, P8, Pz, TP9, TP10, T7, T8, PO3, PO4, PO7, PO8, PO9, PO10, O1, $\mathrm{O} 2, \mathrm{Oz}$ ), using a custom EEG cap with $\mathrm{Ag} / \mathrm{AgCl}$ electrodes and a BrainAmp DC amplifier (Brain Products GmbH, Gilching, Germany). Impedances were kept below $10 \mathrm{k} \Omega$, and referenced to $\mathrm{Cz}$.

For offline processing, Brain Vision Analyzer 2.1.0.1056 (Brain Products $\mathrm{GmbH}$ ) software was used. Data were rereferenced to the average reference across all channels. $\mathrm{Cz}$ was reinstated as an additional channel and data were filtered using $0.1 \mathrm{~Hz}$ high-pass and $20 \mathrm{~Hz}$ lowpass (both $3 \mathrm{~dB}$ point, i.e. half power) Butterworth Zero Phase Filters with $48 \mathrm{~dB}$ /octave roll-off function. The resulting data were segmented into face-locked epochs from -100 to $500 \mathrm{~ms}$. Electrooculogram (EOG) was operationalized by channel pair difference F9 minus F10 for horizontal movements and Fp1 minus left IO for vertical movements.
Exclusion criteria for segments contained erroneous responses and a maximum voltage difference of $100 \mu \mathrm{V}$ for EEG or $60 \mu \mathrm{V}$ for EOG within the whole epoch in order to exclude blinks and eye movements exceeding approximately $4^{\circ}$ of visual angle (Luck \& Kappenman, 2012). The remaining grand average horizontal EOG during the first session did not exceed voltages of $\pm 0.11 \mu \mathrm{V}$ (see Fig. 2a). Subjects were excluded if fewer than 85 epochs remained after artifact rejection for any of the three sessions, leading to exclusion of eleven participants (see Participants). Within the remaining subjects, an average of 219 $(S D=47.3$, range $=89-280)$ out of the 288 trials per session that contained an angry face were kept for further analysis. The number of trials remaining was not affected by session or training condition ( $F s \leq 2.36, p s \geq .096$ ) except for a tendency for subjects in the ABM condition to have an increment of artifact free trials from pre to post training. After averaging the resulting segments for every Subject $\times$ Condition combination, a baseline correction was applied by subtracting the mean amplitude in the time window of -100 to $0 \mathrm{~ms}$ from every data point. A priori, the N2pc was defined to be quantified as the difference between the mean amplitude contralateral minus ipsilateral to the angry face position in the time window $180-300 \mathrm{~ms}$ at electrodes P7/P8 where it is usually maximal (cp. Holmes, Bradley, Kragh Nielsen, \& Mogg, 2009; Luck, 2012; Reutter et al., 2017). A posteriori, we also included a later time window from 300 to $400 \mathrm{~ms}$ because visual inspection of the grand average waveforms indicated effects on the second peak of the N2pc. ${ }^{1}$

\section{Results}

\section{1. $N 2 p c$}

In Fig. 2, a) the grand average waveforms of the N2pc, b) the topographical distributions of its mean amplitudes for both time windows and across the three EEG sessions, and c) mean activations separated by training conditions are shown. The mean amplitude of the first peak $(180-300 \mathrm{~ms})$ of the $\mathrm{N} 2 \mathrm{pc}$ prior to training $(M=-0.25 \mu \mathrm{V}$, $S D=0.52 \mu \mathrm{V}, d=-0.49)$ was statistically smaller than zero $(t$ $(103)=-4.95, p<.001)$ indicating an attentional bias toward angry compared to neutral facial expressions. We calculated a mixed effects ANOVA with the between-subjects factor Training (ABM vs. Dot Probe) and the within-subjects factor Session (pre, post, follow-up). Results revealed that the mean $\mathrm{N} 2 \mathrm{pc}$ amplitude did not change over time $(F(2$, $200)=1.33, p=.267, \eta_{\mathrm{p}}{ }^{2}=0.01$ ) and contrary to our hypotheses no interaction with the training condition occurred $(F(2,200)=0.14$, $\left.p=.869, \eta_{\mathrm{p}}{ }^{2}<0.01\right)$. Lastly, no main effect of Training was found $(F$ $\left.(1,200)=0.22, p=.643, \eta_{\mathrm{p}}{ }^{2}<0.01\right)$. Therefore, the first peak of the $\mathrm{N} 2 \mathrm{pc}$ was not modulated by any factor.

On the second peak (300-400 ms), the attentional bias was even more pronounced before training $(M=-0.33 \mu \mathrm{V}, S D=0.58 \mu \mathrm{V}$, $d=-0.56$ ). Employing the same ANOVA on the mean amplitudes of the later time window, we found a significant main effect of Session ( $F$ $\left.(2,200)=10.19, p<.001, \eta_{\mathrm{p}}{ }^{2}=0.09\right)$ with attentional biases diminishing after training to values that are statistically not different from zero (post: $t(103)=-0.38, p=.352$; follow-up: $t(101)=-1.14$, $p=.129$; both one-tailed). The hypothesized interaction of Session and Training, however, was far from significance $(F(2,200)=0.31$, $\left.p=.735, \eta_{\mathrm{p}}{ }^{2}<0.01\right)$ showing virtually no difference between groups

\footnotetext{
${ }^{1}$ In this article, we tried to be unbiased with respect to the labels attached to the observed event-related lateralization potentials and therefore decided to call both peaks in the early and late time window "N2pc". As noted in the Discussion, the late peak also resembles an ERP known as "SPCN". Wascher and Beste (2010) on the other hand found potentials in similar time frames and called them "N1pc" and "N2pc" for early and late peaks respectively. While we acknowledge that there are good reasons to assign different labels than the ones employed by us, we wanted to focus more on the functionality of the observed potentials and less on the debate around their nomenclature.
} 



b
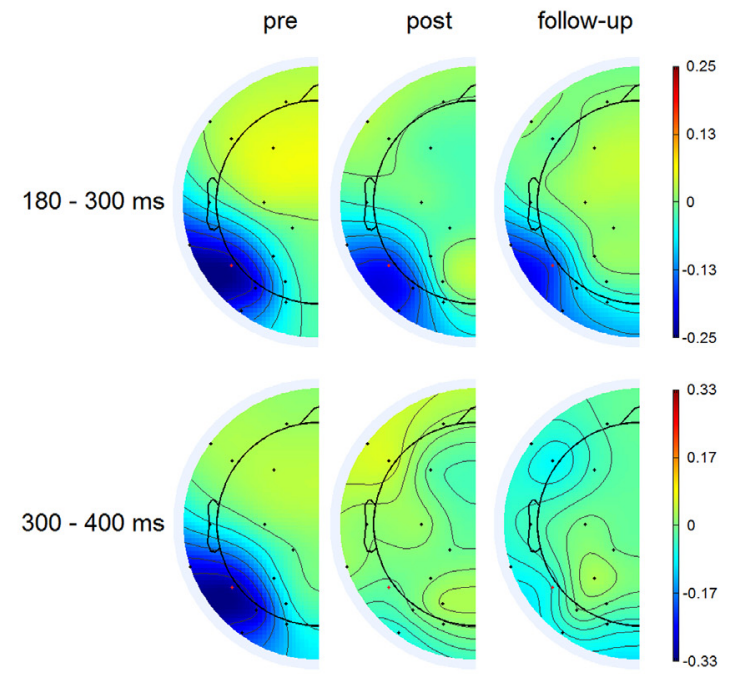

C

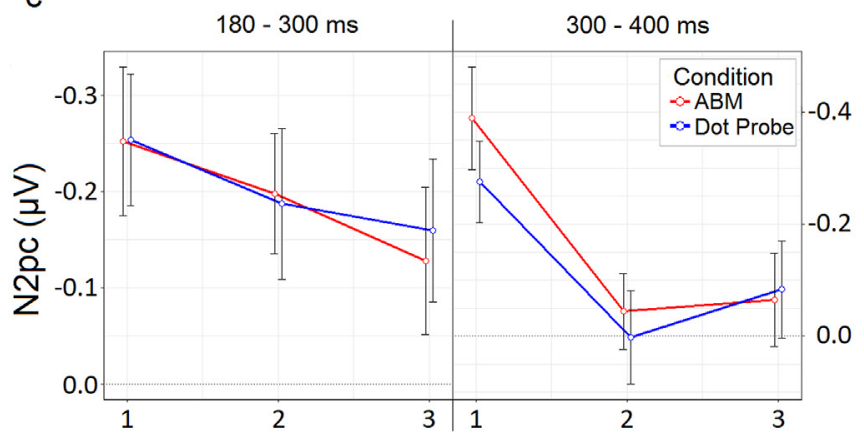

Fig. 2. Development of the N2pc across 3 EEG sessions: pre training, post training, and after 11 weeks follow-up. a) Grand-average waveforms of contraminus ipsilateral to angry face differences at electrodes P7/P8 and remaining horizontal EOG during the first session. b) Topographies for early $(180-300$ $\mathrm{ms})$ and late $(300-400 \mathrm{~ms})$ mean difference potentials. Differences yielded to be maximal at the a priori selected target electrodes P7 and P8. Since values are attained by calculating the difference between homologous electrodes, results have been arbitrarily mapped to the left hemisphere. c) Mean amplitudes of early (180 - $300 \mathrm{~ms})$ and late (300 - $400 \mathrm{~ms})$ difference waves separately for the attentional bias modification (ABM) group and the dot probe control condition. (see Fig. 2c). In addition, no main effect of Training was found $\left(F=0.35, p=.556, \eta_{\mathrm{p}}^{2}<0.01\right)$. We also conducted the same ANOVA but with F9 and F10 as target electrodes in order to control for horizontal eye movements. No effect approached significance (Fs $\leq 0.81$, $p s \geq .371, \eta_{\mathrm{p}}{ }^{2} \mathrm{~s} \leq 0.01$ ). Therefore, the modulation of the N2pc over time is specific for covert attention.

An exploratory analysis including the additional within-subjects factor Distractor Placement (angry face left vs. right) revealed a sizeable main effect $\left(F(1,100)=26.36, p<.001, \eta_{\mathrm{p}}{ }^{2}=0.21\right)$, indicating that angry faces in the left visual hemifield (i.e., processed by the right hemisphere) lead to consistently greater amplitudes across all three time points ( $t s \geq 4.07, p s<.001, d s \geq 0.40$ ) than angry faces in the right half of the visual field. This effect, however, did not interact with other factors ( $\left.F \mathrm{~s} \leq 2.05, p \mathrm{~s} \geq .155, \eta_{\mathrm{p}}{ }^{2} \mathrm{~s} \leq 0.02\right)$ and hence has no influence on the interpretation of the findings reported in the previous analysis.

\subsection{Reaction times}

Responses toward targets co-located with angry faces $(M=574 \mathrm{~ms}$, $S D=58.2 \mathrm{~ms}$ ) did not differ from reaction times to probes in the location of neutral facial expressions $(M=575 \mathrm{~ms}, S D=58.0 \mathrm{~ms})$ in the baseline EEG session $(t(103)=-0.77, \quad p=.221$, one-tailed $)$. Therefore, the RT difference $(M=-0.84 \mathrm{~ms}, \quad S D=11.07 \mathrm{~ms}$, $d=-0.08$ ) was not indicative of an attentional bias with respect to angry or neutral facial expressions. To determine the effect of the training sessions on reaction times, we conducted a mixed effects ANOVA with the RT bias scores as dependent variable, the betweensubjects factor Training (ABM vs. Dot Probe), and the within-subjects factor Session (pre, post, follow-up). Neither a main effect of Session ( $F$ $\left.(2,204)=1.74, p=.179, \eta_{\mathrm{p}}{ }^{2}=0.02\right)$ nor an interaction of Session and Condition $\left(F(2,204)=0.94, p=.392, \eta_{\mathrm{p}}{ }^{2}=0.01\right)$ could be found.

To further investigate the reaction time data, we employed an exploratory mixed effects ANOVA on absolute RTs (instead of partially aggregated RT differences) with the between-subjects factor Training (ABM vs. Dot Probe) and four within-subject factors: Session (pre, post, follow-up), Distractor Placement (angry face left vs. right), Target Category (":" vs. ".. “), and Target Location (left vs. right). This analysis revealed a huge main effect of Session $(F(2,204)=337.18, p<.001$, $\left.\eta_{\mathrm{p}}{ }^{2}=0.77\right)$ with reaction times significantly decreasing after training $(\Delta=-98 \mathrm{~ms}, d=-2.24, t(103)=-22.85, p<.001)$ and slightly declining further until the follow-up session $(\Delta=-14 \mathrm{~ms}, d=-0.40$, $t(103)=-4.04, p<.001)$. We also found a main effect of Target Location $\left(F(1,102)=48.98, p<.001, \eta_{\mathrm{p}}{ }^{2}=0.32\right)$ indicating faster responses to targets in the right visual hemifield ( $\Delta=9 \mathrm{~ms}, d=0.69, t$ $(103)=7.03, p<.001)$. Two highly surprising effects also reached significance: An interaction of Training with Distractor Placement on the one hand $\left(F(1,102)=8.52, p=.004, \eta_{\mathrm{p}}{ }^{2}=0.08\right)$ and an interaction of Training with Target Category $(F(1,102)=3.98, p=.049$, $\eta_{\mathrm{p}}{ }^{2}=0.04$ ) on the other hand. Visual inspection of the first effect revealed that only for angry faces in the right visual hemifield, differences between training conditions occurred: Participants who received ABM reacted faster to targets than control subjects (independent of target location or category). The second interaction was due to individuals in the control condition reacting faster to upright colons while subjects in the ABM group were faster when rotated colons occurred. Since the two training conditions were identical for the first EEG recording and both effects did not interact with Session ( $F s \leq 2.43, p s \geq .093, \eta_{\mathrm{p}}{ }^{2} \mathrm{~s} \leq 0.05$ ), however, they are likely due to randomization and were not further interpreted. No other effect reached significance ( $F \mathrm{~s} \leq 3.02, p \mathrm{~s} \geq .069$, $\left.\eta_{\mathrm{p}}{ }^{2} \mathrm{~s} \leq 0.03\right)$.

\subsection{Questionnaires}

For all employed questionnaires, we conducted mixed effects ANOVAs with the between-subjects factor Training (ABM vs. Dot 
Probe) and the within-subjects factor Session (pre, post, follow-up). Correlations between self-reports and measures of biased attention at baseline have indicated that attentional biases toward socially threatening stimuli are specifically related to symptoms of social anxiety in a previous report (Reutter et al., 2017).

Anxiety Symptoms. All three questionnaires targeting social anxiety (SIAS, SPAI, and SPS) were unaffected by our experimental design ( $F \mathrm{~s} \leq 1.20, p s \geq .301, \eta_{\mathrm{p}}{ }^{2} \mathrm{~s} \leq 0.01$ ). The same is true for general anxiety measured with the STAI $\left(F s \leq 0.84, p s \geq .424, \eta_{\mathrm{p}}{ }^{2} \mathrm{~s} \leq 0.01\right.$ ). Consequently, neither the ABM procedure nor the control condition exhibited changes on anxiety symptoms.

Depressive Symptoms. We did find, however, an interaction of Training and Session on depressive symptomatology as indexed by the BDI-V $\left(F(2,218)=4.19, p=.021, \eta_{\mathrm{p}}{ }^{2}=0.04\right)$. This effect was mostly due to a decrease in reported depressive symptoms from post to followup for participants in the ABM condition $(t(54)=3.11, p=.003)$ while control subjects showed a marginally significant decrease already from pre to post training $(t(55)=1.82, p=.075)$.

Personality Traits. Finally, we assessed changes in personality traits with the BFAS scale. We found a main effect of Session on the neuroticism subscale $\left(F(2,218)=5.04, \quad p=.009, \quad \eta_{\mathrm{p}}{ }^{2}=0.04\right)$. Individuals showed a decrease in neuroticism from pre to post training regardless of training condition $(t(110)=2.65, p=.009)$. These changes remained stable until the follow-up assessment $(t(110)=0.64$, $p=.521$ ). No significant effects were found for Agreeableness, Conscientiousness, Extraversion, and Openness/Intellect ( Fs $\leq 3.09$, $\left.p s \geq .056, \eta_{\mathrm{p}}^{2} \mathrm{~s} \leq 0.03\right)$.

\subsection{Reliabilities}

To determine reliability of our measurements, we calculated correlation coefficients of odd and even trials for both the N2pc and reaction times. In order to account for test bisection we applied the Spearman-Brown prophecy correction. For questionnaire data, we employed Cronbach's alpha.

N2pc. The N2pc before training showed unacceptable reliability for both early $(r=0.25)$ and late $(r=0.39)$ peaks. Since we found differential effects for angry faces in either visual hemifield in an explorative analysis, we also calculated reliability indices for these conditions separately, splitting the N2pc into two subscores based on the location of the angry facial stimulus. After separation by hemifield, the N2pc subscores displayed excellent reliability for both time windows and for both the first and second EEG sessions ( $r \leq 0.91$ ). Only during the follow-up assessment, reliability dropped to merely acceptable values for early N2pc subscores ( $r=0.77$ for both hemifields) while in the late time window it was still excellent for angry faces in the right visual hemifield $(r=0.94)$ and good for angry faces on the left half of the screen $(r=0.83)$.

RTs. The reliability for the RT bias during the first EEG assessment was unacceptably low $(r=0.21)$. Conversely, the absolute reaction times toward probes co-located with angry or neutral facial expressions, i.e., the components that constitute this difference score, showed almost perfect reliability ( $r=0.98$ for both distractor categories). Since we found an effect of target location, we also assessed the reliability of the RT difference toward targets on the left minus right half of the screen. This bias toward the right visual hemifield showed acceptable reliability $(r=0.73)$.

Questionnaires. Cronbach's alpha coefficients indicated excellent reliability for most of the employed questionnaires across all three EEG sessions (STAI: $\alpha$ s $\geq 0.89$; SIAS: $\alpha s \geq 0.90$; SPAI: $\alpha s \geq 0.96$; SPS: as $\geq 0.91$; BDI: $\alpha s \geq 0.91$ ). For the BFAS, reliability was good for all subscales across all three assessments (Neuroticism: $\alpha \mathrm{s} \geq 0.88$; Extraversion: $\alpha s \geq 0.84$; Openness/Intellect: $\alpha \mathrm{s} \geq 0.79$; Agreeableness: as $\geq 0.86$; Conscientiousness: $\alpha \mathrm{s} \geq 0.82$ ).

\section{Discussion}

In the present study, we assessed the effect of an extensive attentional bias modification (ABM) procedure, consisting of close to 7000 trials, concurrently with behavioral and electrophysiological measures within a large sample of over 100 highly socially anxious participants. Results showed that the N2pc component demonstrates more internal consistency and more statistical power in detecting attentional biases and their modification than reaction time differences. RT bias scores neither indicated an attentional preference for angry compared to neutral facial expressions before training nor a modification of this relation over time. Conversely, the N2pc event-related lateralization clearly indexed an attentional bias at baseline and also revealed an effect of the training sessions, however only on the second peak between 300 and $400 \mathrm{~ms}$.

Our results indicated that there was no difference between an active ABM condition (i.e., 100\% contingency of targets away from threat) and a dot probe control providing mere exposition to the stimuli. Both groups showed similar changes over time (or, in case of RT differences, a lack thereof). A recent review by Mogg and collaborators (2017) demonstrated that control procedures which incorporate some kind of attention training oftentimes elicit similar changes as threat-avoidance tasks. Additionally, a study by Heeren, Coussement, and McNally (2016) cast doubt on whether emotional content is necessary at all for attentional bias modification: They employed similar training conditions but with non-emotional geometric shapes as distractors and another control condition completely without distractor stimuli. They also did not find any differences between groups. Hence, attentional bias modification procedures might not depend on contingencies or not even on threat-relevant content, casting concerns on the theoretical underpinnings of $\mathrm{ABM}$ literature.

Another possibility is that other variants of $A B M$ protocols than the one we employed possess greater differential efficacy (cp. Mogg \& Bradley, 2018). When data acquisition for this longitudinal study started, it was recommended to not be explicit about the purpose of the ABM training (Bar-Haim, 2010; MacLeod, Mackintosh, \& Vujic, 2009) but recently this has been revised by both empirical (Nishiguchi, Takano, \& Tanno, 2015) and theoretical accounts (Mogg \& Bradley, 2018). Furthermore, while eight ABM sessions seemed to be advisable in the past to maximize behavioral training effects (Amir et al., 2009; Bar-Haim, 2010; Schmidt, Richey, Buckner, \& Timpano, 2009), motivational aspects would indicate that trainings ought not to be designed too repetitively (Mogg \& Bradley, 2018). Lastly, there has also been controversy about the amount of cognitive load that should be imposed on participants (Booth, Mackintosh, Mobini, Oztop, \& Nunn, 2014; Clarke et al., 2017). Future research should further evaluate the efficacy of different parametrization of ABM protocols.

An important question is why we found an attentional preference for angry compared to neutral facial expressions for both time windows of the N2pc but an alteration across sessions was only present at later stages of processing. One explanation is that $\mathrm{ABM}$ and similar exposition trainings do not target the primary visual cortex directly but influence it indirectly via connections from the amygdala and the prefrontal cortex (PFC). Recent work has demonstrated that hyperactivity in the amygdala plays an important role in the processing of negative emotional content (Etkin \& Wager, 2007) and that ABM procedures induce down-regulation of this activity (Taylor et al., 2014). Fittingly, subjects with higher baseline activity in the left amygdala benefit more from ABM (Britton et al., 2015). The prefrontal cortex can modulate amygdalar hyperreactivity to threat but fails to do so when anxiety becomes clinical (Monk et al., 2008). Cognitive behavioral therapy and serotonin specific reuptake inhibitors help the PFC to engage in response to threatening stimuli (Maslowsky et al., 2010). Similar upregulations of the PFC have been observed for ABM procedures (Taylor et al., 2014). Moreover, anodal transcranial direct current stimulation of the dorsolateral prefrontal cortex actively facilitates ABM in healthy 
(Clarke, Browning, Hammond, Notebaert, \& MacLeod, 2014; Heeren, Baeken, Vanderhasselt, Philippot, \& Raedt, 2015) and clinical subjects (Heeren et al., 2017). Since threat processing is subject to modulations by the PFC and the amygdala, and ABM induces compensatory changes in these brain regions, phasic modulation of the visual cortex might only occur after a few hundred milliseconds.

What is the functionality of these two peaks of event-related lateralization? In a related paradigm, Wascher and Beste (2010) found similar grand average waveforms and claimed that the earlier peak indexes a first processing step of information competition and weighting while the second peak is indicative of reallocation after an initial (and possibly biased) perception. Consequently, our results could imply that our manipulations did not modify initial processing but encouraged attentional reallocation away from threat, diminishing the temporal persistency of biased perception. Some researchers have labeled N2pclike lateralizations starting at $300 \mathrm{~ms}$ post stimulus onset as sustained posterior contralateral negativity (SPCN; Robitaille \& Jolicœur, 2006). This potential has been linked to stimulus encoding into visual shortterm memory (Prime, Pluchino, Eimer, Dell'Acqua, \& Jolicœur, 2011). Hence, the outcome of this study could also be interpreted as reduced encoding after repeated exposition to the stimuli and would partially explain the lack of group differences between ABM and control condition. The SPCN, however, has not only been interpreted as stimulus encoding but also as internal reprocessing of a stimulus (Schneider, Hoffmann, \& Wascher, 2014) which in turn could drive the attentional reallocation process described before. Clark, Appelbaum, van den Berg, Mitroff, and Woldorff (2015) interpreted a decreased SPCN after practice as facilitated stimulus discrimination but they also found earlier and larger amplitudes of the N2pc that we didn't observe.

We found hemispheric asymmetries in both our electrophysiological and behavioral data. For the N2pc, our results might reflect the right hemisphere's specialization for visuospatial processing (Gazzaniga, 2005). It is also possible, however, that the hemispheric differences in electrophysiological activation that we observed reflect mere structural asymmetries that have no functional implication for covert attention (Toga \& Thompson, 2003). More specifically, our findings converge to work on other face-specific ERP components like the N170 with respect to dominance of the right hemisphere, i.e., the left visual hemifield (Karl, Hewig, \& Osinsky, 2016; Kolassa \& Miltner, 2006; Mogg \& Bradley, 2002; Rossion, Joyce, Cottrell, \& Tarr, 2003; Wieser et al., 2018; but see Carlson \& Reinke, 2010; Rossignol, Campanella, Bissot, \& Philippot, 2013 for opposite patterns). The RT data, however, indicated preference of the right visual field (see also Brosch, Pourtois, Sander, \& Vuilleumier, 2011). Therefore, if the N2pc hemispheric differences were to be interpreted in a functional way, it would indicate an opposite pattern to the RT data within the same sample. This controversy can be resolved by considering the visual elements that both variables are based upon: The N2pc emerges as a consequence of the visual processing of the facial distractor stimuli, whereas reaction times are a behavioral result of the visual processing of the dot target. Ossandón, Onat, and König (2014) have shown that in German right-handers, attention deployed to visual scenes shows an initial bias toward the left visual field and slightly shifts to the right afterward. This pattern might be subject to cultural differences in reading direction (Eviatar, 1995). Likewise in our experiment, attention might have been deployed more strongly to the left side initially while distractors were shown and then cycled to the right when targets emerged. When Mogg and Bradley (1999) applied a Dot Probe Paradigm with a stimulus onset asynchrony (SOA) between distractors and targets as little as $14 \mathrm{~ms}$, however, reaction to probes captured the initial bias toward objects in the left visual field. The time frame between distractors and targets might therefore be a crucial determinant whether the N2pc and RT differences capture the same construct (cp. Reutter et al., 2017).

These hemispheric differences are not a mere technicality but have important consequences for psychometric quality. Considering the reliability estimates of the N2pc, they seem to play a vital role. Following the standard protocol of the N2pc, i.e., collapsing across angry faces processed in the left and right visual hemifield, lead to unacceptably low reliability ( $r s \leq 0.39$ ). Conversely, when calculated for angry faces in either hemisphere separately, reliability of these N2pc subscores was excellent ( $r s \geq 0.91$ ). This indicates that the N2pc as an electrophysiological marker can only bear validity if hemispheric differences are accounted for.

The reaction time data in our experiment were not indicative of attentional biases at baseline or of an alteration after training. Due to our preselection of highly socially anxious individuals, however, the presence of attentional biases should be expected (Bar-Haim et al., 2007). One explanation is the lack of reliability associated with RT differences (Dear et al., 2011; Price et al., 2015; Reutter et al., 2017; Rodebaugh et al., 2016; Schmukle, 2005; Staugaard, 2009; Van Bockstaele et al., 2011; Waechter et al., 2014; Waechter \& Stolz, 2015) that was replicated with our data. Another reason, however, is that we optimized the experimental setting with respect to the N2pc component: Firstly, we prohibited saccades during the whole trial because the $\mathrm{N} 2 \mathrm{pc}$ only works with covert attention. Secondly, in order to not confound the ERP with the visual onset of the probe, we selected a relatively high SOA between distractors and targets of $500 \mathrm{~ms}$. To our knowledge, the role of overt attention in the Dot Probe has never been systematically investigated. In search tasks, in which the N2pc is predominantly utilized, deployment of covered attention is usually sufficient to evoke RT differences (Feldmann-Wüstefeld et al., 2011; Weymar et al., 2011; Weymar, Gerdes, Löw, Alpers, \& Hamm, 2013). The Dot Probe, however, requires reactions to different stimuli than the ones that guide attention (i.e., probes and distractors respectively). Therefore, it might be necessary to deploy overt attention such that targets appear more frequently at already fixated locations in order for a reliable difference in reaction times to occur. In accordance with this interpretation, recent research combining the N2pc and the Dot Probe Paradigm frequently did not find reaction time biases (Kappenman et al., 2014; Kappenman et al., 2015; Osinsky et al., 2014; but see; Holmes et al., 2009; Holmes, Mogg, Fockert, Nielsen, \& Bradley, 2014). Brosch et al. (2011) also prohibited eye movements and they only consistently found significant RT biases during short SOAs $(50 \mathrm{~ms})$ compared to long intervals $(750 \mathrm{~ms})$.

With respect to SOAs, a recent meta-analysis of facial Dot Probe Paradigms with socially anxious participants suggested that they correlate negatively with RT biases such that with an asynchrony of $500 \mathrm{~ms}$ attentional biases can barely be revealed (Bantin, Stevens, Gerlach, \& Hermann, 2016). Torrence, Wylie, and Carlson (2017) even found a threshold as low as $300 \mathrm{~ms}$. Mechanistically, the moderating influence of SOA on attentional biases may be caused by cycles of attentional engagement and disengagement between both distractors, with any trial-level reaction time mapping to the current attentional state right before target onset. For larger SOAs, several cycles may have already occurred prior to the revelation of the probe such that the current state of vigilance to or avoidance of threat is best described by a coin flip, rendering mean RT differences as not diagnostic for attentional biases. For small SOAs, however, RTs may predominantly capture the first cycle of attentional deployment, providing a better indicator of biased attention.

Our data indicates that attentional biases were successfully shifted away from threat but what about anxiety symptomatology? Unfortunately, changes in self-reported symptoms were found neither on general trait anxiety nor on social anxiety even though we used a procedure that has been shown to reliably invoke such modifications (MacLeod \& Grafton, 2016). Several reasons might be responsible for this. According to the review of meta-analyses by Jones and Sharpe (2017), ABM is superior in providing a buffer to stressor vulnerability while interpretative bias modification serves better as a means to symptom reduction. However, we have not measured stress reactivity in our study since they are usually employed in single session ABM protocols (Clarke, MacLeod, \& Notebaert, 2014). Moreover, utilizing 
words instead of pictures as distractor stimuli seems to also yield better outcomes for symptom reduction (Jones \& Sharpe, 2017). Lastly, selfreport measures are considered inferior with respect to their sensitivity as compared to external ratings by a trained clinician (Jones \& Sharpe, 2017; Price et al., 2016). While we didn't find a change in anxiety symptomatology, we did observe an effect on trait neuroticism. Subjects reported a decrease after training that remained stable until 11 weeks later. Neuroticism is one of the main personality dimensions according to many different theories of personality (Costa \& McCrae, 2008; Eysenck, 1990; Gray \& McNaughton, 1996) that often includes anxiety as a subdomain (Costa \& McCrae, 2008) or as a closely related theoretical construct (e.g., Gray, 1970; Gray \& McNaughton, 1996).

Lastly, we found an unexpected decline in self-reported depressive symptoms that emerged during the waiting period prior to the followup session in the ABM group. Since no manipulation was administered in the meantime, we expect this change to be driven by uncontrolled events during these 11 weeks. Our screening did not yield to be specific for elevated social anxiety but also preselected subjects with heightened depressive symptomatology as evident by sizeable intercorrelations between the BDI-V and all questionnaires targeting social anxiety ( $r s \geq 0.508, p s<.001$ ). Subjects with mild, undiagnosed depression might then have experienced remission during the waiting period. In the future, screening procedures should be tuned more carefully in order to be specific for the target symptomatology without confounding comorbid symptoms.

To summarize, electrophysiological measures are an important tool to assess attentional biases and their modification with excellent reliability. Methodological pitfalls like hemispheric influences, however, need to be addressed to ensure psychometric quality. In the future, the N2pc should be evaluated in a clinical sample with respect to attentional biases and their modification. Another promising option is an explicit reward system to further enhance attentional bias modification (Sigurjónsdóttir, Björnsson, Ludvigsdóttir, \& Kristjánsson, 2015) especially since the N2pc is modulated by reward (Kiss, Driver, \& Eimer, 2009).

\section{Author note}

This work was supported by the German Research Foundation (DFG fund numbers HE 5330/8-1, OS 422/4-1, and WI 2714/7-1).

We are grateful to Manuel Blickle, Simone Neurath, Merle Rasch, Natalie-Isabell Zastrow, Inse Janssen, Barbara Winter, Olivia Müller, Ann-Kathrin Mittnacht, and Anna-Lina Blank for their assistance in data collection.

\section{Appendix A}

The data base reference is: Reutter, M. (2019, May 21). N2pc Attentional Bias Modification. https://doi.org/10.17605/OSF.IO/ SP2XY (the repository is hosted at the Open Science Foundation repository osf.io).

\section{References}

Ahrens, L. M., Mühlberger, A., Pauli, P., \& Wieser, M. J. (2015). Impaired visuocortical discrimination learning of socially conditioned stimuli in social anxiety. Social Cognitive and Affective Neuroscience, 10, 929-937. https://doi.org/10.1093/scan/ nsu140.

Amir, N., Beard, C., Taylor, C. T., Klumpp, H., Elias, J., Burns, M., et al. (2009). Attention training in individuals with generalized social phobia: A randomized controlled trial. Journal of Consulting and Clinical Psychology, 77, 961-973. https://doi.org/10.1037/ a0016685.

Bantin, T., Stevens, S., Gerlach, A. L., \& Hermann, C. (2016). What does the facial dotprobe task tell us about attentional processes in social anxiety? A systematic review. Journal of Behavior Therapy and Experimental Psychiatry, 50, 40-51. https://doi.org/ 10.1016/j.jbtep.2015.04.009.

Bar-Haim, Y. (2010). Research review: Attention bias modification (ABM): A novel treatment for anxiety disorders. The Journal of Child Psychology and Psychiatry and Allied Disciplines, 51, 859-870. https://doi.org/10.1111/j.1469-7610.2010.02251.x.
Bar-Haim, Y., Lamy, D., Pergamin, L., Bakermans-Kranenburg, M. J., \& van IJzendoorn, M. H. (2007). Threat-related attentional bias in anxious and nonanxious individuals: A meta-analytic study. Psychological Bulletin, 133, 1-24. https://doi.org/10.1037/ 0033-2909.133.1.1.

Beck, A. T., Steer, R. A., \& Brown, G. K. (1996). Beck depression inventory-II, Vol. 78, 490-498 San Antonio.

Booth, R. W., Mackintosh, B., Mobini, S., Oztop, P., \& Nunn, S. (2014). Cognitive bias modification of attention is less effective under working memory load. Cognitive Therapy and Research, 38, 634-639. https://doi.org/10.1007/s10608-014-9628-6.

Bradley, M. M., \& Lang, P. J. (1994). Measuring emotion: The self-assessment manikin and the semantic differential. Journal of Behavior Therapy and Experimental Psychiatry, 25, 49-59. https://doi.org/10.1016/0005-7916(94)90063-9.

Britton, J. C., Suway, J. G., Clementi, M. A., Fox, N. A., Pine, D. S., \& Bar-Haim, Y. (2015). Neural changes with attention bias modification for anxiety: A randomized trial. Social Cognitive and Affective Neuroscience, 10, 913-920. https://doi.org/10.1093/ scan/nsu141.

Brosch, T., Pourtois, G., Sander, D., \& Vuilleumier, P. (2011). Additive effects of emotional, endogenous, and exogenous attention: Behavioral and electrophysiological evidence. Neuropsychologia, 49, 1779-1787. https://doi.org/10.1016/j. neuropsychologia.2011.02.056.

Carlson, J. M., \& Reinke, K. S. (2010). Spatial attention-related modulation of the N170 by backward masked fearful faces. Brain and Cognition, 73, 20-27. https://doi.org/ 10.1016/j.bandc. 2010.01.007.

Clark, K., Appelbaum, L. G., van den Berg, B., Mitroff, S. R., \& Woldorff, M. G. (2015). Improvement in visual search with practice: Mapping learning-related changes in neurocognitive stages of processing. Journal of Neuroscience : The Official Journal of the Society for Neuroscience, 35, 5351-5359. https://doi.org/10.1523/JNEUROSCI 1152-14.2015.

Clarke, P. J. F., Branson, S., Chen, N. T. M., van Bockstaele, B., Salemink, E., MacLeod, C., et al. (2017). Attention bias modification training under working memory load increases the magnitude of change in attentional bias. Journal of Behavior Therapy and Experimental Psychiatry, 57, 25-31. https://doi.org/10.1016/j.jbtep.2017.02.003.

Clarke, P. J. F., Browning, M., Hammond, G., Notebaert, L., \& MacLeod, C. (2014). The causal role of the dorsolateral prefrontal cortex in the modification of attentional bias: Evidence from transcranial direct current stimulation. Biological Psychiatry, 76, 946-952. https://doi.org/10.1016/j.biopsych.2014.03.003.

Clarke, P. J. F., MacLeod, C., \& Notebaert, L. (2014). Absence of evidence or evidence of absence: Reflecting on therapeutic implementations of attentional bias modification. BMC Psychiatry, 14, 8. https://doi.org/10.1186/1471-244X-14-8.

Costa, P. T., \& McCrae, R. R. (2008). The NEO inventories. In R. P. Archer, \& S. R. Smith (Eds.). Personality assessment (pp. 213-245). New York: Taylor \& Francis.

Dear, B. F., Sharpe, L., Nicholas, M. K., \& Refshauge, K. (2011). The psychometric properties of the dot-probe paradigm when used in pain-related attentional bias research. The Journal of Pain : Official Journal of the American Pain Society, 12, 1247-1254. https://doi.org/10.1016/j.jpain.2011.07.003.

DeYoung, C. G., Quilty, L. C., \& Peterson, J. B. (2007). Between facets and domains: 10 aspects of the Big five. Journal of Personality and Social Psychology, 93, 880-896. https://doi.org/10.1037/0022-3514.93.5.880.

Eimer, M. (1996). The N2pc component as an indicator of attentional selectivity. Electroencephalography and Clinical Neurophysiology, 99, 225-234. https://doi.org/10. 1016/0013-4694(96)95711-9.

Eimer, M., \& Kiss, M. (2007). Attentional capture by task-irrelevant fearful faces is revealed by the N2pc component. Biological Psychology, 74, 108-112. https://doi.org/ 10.1016/j.biopsycho.2006.06.008.

Etkin, A., \& Wager, T. D. (2007). Functional neuroimaging of anxiety: A meta-analysis of emotional processing in PTSD, social anxiety disorder, and specific phobia. American Journal of Psychiatry, 164, 1476-1488. https://doi.org/10.1176/appi.ajp.2007. 07030504.

Eviatar, Z. (1995). Reading direction and attention: Effects on lateralized ignoring. Brain and Cognition, 29, 137-150. https://doi.org/10.1006/brcg.1995.1273.

Eysenck, H. J. (1990). Biological dimensions of personality. In L. A. Pervin (Ed.). Handbook of personality: Theory and research. Guilford Publications.

Feldmann-Wüstefeld, T., Schmidt-Daffy, M., \& Schubö, A. (2011). Neural evidence for the threat detection advantage: Differential attention allocation to angry and happy faces. Psychophysiology, 48, 697-707. https://doi.org/10.1111/j.1469-8986.2010. 01130.x.

Fenker, D. B., Heipertz, D., Boehler, C. N., Schoenfeld, M. A., Noesselt, T., Heinze, H.-J., ... Hopf, J.-M. (2010). Mandatory processing of irrelevant fearful face features in visual search. Journal of Cognitive Neuroscience, 22, 2926-2938. https://doi.org/10.1162/ jocn. 2009.21340.

Fox, E., Derakshan, N., \& Shoker, L. (2008). Trait anxiety modulates the electrophysiological indices of rapid spatial orienting towards angry faces. NeuroReport, 19, 259-263. https://doi.org/10.1097/WNR.0b013e3282f53d2a.

Fydrich, T., Scheurich, A., \& Kasten, E. (1995). Fragebogen zur sozialen Angst; deutsche Bearbeitung des Social Phobia and Anxiety Inventory (SPAI) von Turner und Beidel. Psychologisches Institut Der Universität Heidelberg.

Gazzaniga, M. S. (2005). Forty-five years of split-brain research and still going strong. Nature Reviews Neuroscience, 6(8), 653. https://doi.org/10.1038/nrn1723.

Goeleven, E., Raedt, R. de, Leyman, L., \& Verschuere, B. (2008). The Karolinska directed emotional faces: A validation study. Cognition \& Emotion, 22, 1094-1118. https://doi. org/10.1080/02699930701626582.

Gray, J. A. (1970). The psychophysiological basis of introversion-extraversion. Behaviour Research and Therapy, 8, 249-266. https://doi.org/10.1016/0005-7967(70)90069-0.

Gray, J. A., \& McNaughton, N. (1996). The neuropsychology of anxiety: Reprise. In D. A. Hope, \& C. E. Izard (Vol. Eds.), Current theory and research in motivation,: Vol. 43, (pp. 61-134). Lincoln, NE, US: University of Nebraska Press Perspectives on Anxiety, 
Panic, and Fear.

Heeren, A., Baeken, C., Vanderhasselt, M.-A., Philippot, P., \& Raedt, R. de (2015). Impact of anodal and cathodal transcranial direct current stimulation over the left dorsolateral prefrontal cortex during attention bias modification: An eye-tracking study. PLoS One, 10, e0124182. https://doi.org/10.1371/journal.pone.0124182.

Heeren, A., Billieux, J., Philippot, P., Raedt, R. de, Baeken, C., Timary, P. de, . ., et al. (2017). Impact of transcranial direct current stimulation on attentional bias for threat: A proof-of-concept study among individuals with social anxiety disorder. Social Cognitive and Affective Neuroscience, 12, 251-260. https://doi.org/10.1093/ scan/nsw119.

Heeren, A., Coussement, C., \& McNally, R. J. (2016). Untangling attention bias modification from emotion: A double-blind randomized experiment with individuals with social anxiety disorder. Journal of Behavior Therapy and Experimental Psychiatry, 50 , 61-67. https://doi.org/10.1016/j.jbtep.2015.05.005.

Holmes, A., Bradley, B. P., Kragh Nielsen, M., \& Mogg, K. (2009). Attentional selectivity for emotional faces: Evidence from human electrophysiology. Psychophysiology, 46, 62-68. https://doi.org/10.1111/j.1469-8986.2008.00750.x.

Holmes, A., Mogg, K., Fockert, J. de, Nielsen, M. K., \& Bradley, B. P. (2014) Electrophysiological evidence for greater attention to threat when cognitive control resources are depleted. Cognitive, Affective, \& Behavioral Neuroscience, 14, 827-835. https://doi.org/10.3758/s13415-013-0212-4.

Jones, E. B., \& Sharpe, L. (2017). Cognitive bias modification: A review of meta-analyses. Journal of Affective Disorders, 223, 175-183. https://doi.org/10.1016/j.jad.2017.07. 034.

Kappenman, E. S., Farrens, J. L., Luck, S. J., \& Proudfit, G. H. (2014). Behavioral and ERP measures of attentional bias to threat in the dot-probe task: Poor reliability and lack of correlation with anxiety. Frontiers in Psychology, 5, 1368. https://doi.org/10.3389/ fpsyg.2014.01368.

Kappenman, E. S., MacNamara, A., \& Proudfit, G. H. (2015). Electrocortical evidence for rapid allocation of attention to threat in the dot-probe task. Social Cognitive and Affective Neuroscience, 10, 577-583. https://doi.org/10.1093/scan/nsu098.

Karl, C., Hewig, J., \& Osinsky, R. (2016). Passing faces: Sequence-dependent variations in the perceptual processing of emotional faces. Social Neuroscience, 11, 531-544. https://doi.org/10.1080/17470919.2015.1115776.

Kessler, R. C., Berglund, P., Demler, O., Jin, R., Merikangas, K. R., \& Walters, E. E. (2005). Lifetime prevalence and age-of-onset distributions of DSM-IV disorders in the national comorbidity survey replication. Archives of General Psychiatry, 62, 593-602. https://doi.org/10.1001/archpsyc.62.6.593.

Kiss, M., Driver, J., \& Eimer, M. (2009). Reward priority of visual target singletons modulates event-related potential signatures of attentional selection. Psychological Science, 20, 245-251. https://doi.org/10.1111/j.1467-9280.2009.02281.x.

Kiss, M., van Velzen, J., \& Eimer, M. (2008). The N2pc component and its links to attention shifts and spatially selective visual processing. Psychophysiology, 45, 240-249. https://doi.org/10.1111/j.1469-8986.2007.00611.x.

Kolassa, I.-T., \& Miltner, W. H. R. (2006). Psychophysiological correlates of face processing in social phobia. Brain Research, 1118, 130-141. https://doi.org/10.1016/j brainres.2006.08.019.

Koster, E. H. W., Crombez, G., Verschuere, B., \& Houwer, J. de (2004). Selective attention to threat in the dot probe paradigm: Differentiating vigilance and difficulty to disengage. Behaviour Research and Therapy, 42, 1183-1192. https://doi.org/10.1016/j. brat.2003.08.001.

Laux, L., Glanzmann, P., Schaffner, P., \& Spielberger, C. D. (1981). Das state-trait-anxietyinventory (STAI). Theoretical foundations and instructions.

Luck, S. J. (2012). Electrophysiological correlates of the focusing of attention within complex visual scenes: N2pc and related ERP components. In S. J. Luck, \& E. S. Kappenman (Eds.). The oxford handbook of event-related potential components (pp. 329360). New York: Oxford University Press.

Luck, S. J., \& Hillyard, S. A. (1994). Electrophysiological correlates of feature analysis during visual search. Psychophysiology, 31, 291-308. https://doi.org/10.1111/j. 1469-8986.1994.tb02218.x.

Luck, S. J., \& Kappenman, E. S. (Eds.). (2012). The oxford handbook of event-related potential components. New York: Oxford University Press.

Lundqvist, D., Flykt, A., \& Öhman, A. (1998). The Karolinska directed emotional faces (KDEF). CD ROM from Department of Clinical Neuroscience, Psychology Section, Karolinska Institutet.

MacLeod, C., \& Grafton, B. (2016). Anxiety-linked attentional bias and its modification Illustrating the importance of distinguishing processes and procedures in experimental psychopathology research. Behaviour Research and Therapy, 86, 68-86. https://doi.org/10.1016/j.brat.2016.07.005.

MacLeod, C., Mackintosh, B., \& Vujic, T. (Eds.). (2009). Does the explicit communication of the training contingency enhance the efficacy of cognitive behavior modification (CBM).

MacLeod, C., Mathews, A. \& Tata, P. (1986). Attentional bias in emotional disorders. Journal of Abnormal Psychology, 95, 15-20. https://doi.org/10.1037//0021-843X.95. 1.15 .

Maslowsky, J., Mogg, K., Bradley, B. P., McClure-Tone, E., Ernst, M., Pine, D. S., et al. (2010). A preliminary investigation of neural correlates of treatment in adolescents with generalized anxiety disorder. Journal of Child and Adolescent Psychopharmacology, 20, 105-111. https://doi.org/10.1089/cap.2009.0049.

Mathews, A., \& MacLeod, C. (2002). Induced processing biases have causal effects on anxiety. Cognition \& Emotion, 16, 331-354. https://doi.org/10.1080/ 02699930143000518.

Mattick, R. P., Clarke, J. C., \& S.T (1998). Development and validation of measures of social phobia scrutiny fear and social interaction anxiety11Editor's note: This article was written before the development of some contemporary measures of social phobia, such as the Social Phobia and Anxiety Inventory (Turner et al., 1989). We have invited this article for publication because of the growing interest in the scales described therein. Behaviour Research and Therapy, 36, 455-470. https://doi.org/10. 1016/S0005-7967(97)10031-6.

Mogg, K., \& Bradley, B. P. (1999). Orienting of attention to threatening facial expressions presented under conditions of restricted awareness. Cognition \& Emotion, 13, 713-740. https://doi.org/10.1080/026999399379050.

Mogg, K., \& Bradley, B. P. (2002). Selective orienting of attention to masked threat faces in social anxiety. Behaviour Research and Therapy, 40, 1403-1414. https://doi.org/10. 1016/S0005-7967(02)00017-7.

Mogg, K., \& Bradley, B. P. (2018). Anxiety and threat-related attention: Cognitive-motivational framework and treatment. Trends in Cognitive Sciences, 22, 225-240. https:// doi.org/10.1016/j.tics.2018.01.001.

Mogg, K., Waters, A. M., \& Bradley, B. P. (2017). Attention bias modification (ABM): Review of effects of multisession ABM training on anxiety and threat-related attention in high-anxious individuals. Clinical Psychological Science : A Journal of the Association for Psychological Science, 5, 698-717. https://doi.org/10.1177/ 2167702617696359.

Monk, C. S., Telzer, E. H., Mogg, K., Bradley, B. P., Mai, X., Louro, H. M. C., ... Pine, D. S. (2008). Amygdala and ventrolateral prefrontal cortex activation to masked angry faces in children and adolescents with generalized anxiety disorder. Archives of General Psychiatry, 65, 568-576. https://doi.org/10.1001/archpsyc.65.5.568.

Mussel, P., \& Paelecke, M. (in prep.). Reliabilität und Validität einer deutschsprachigen Fassung der Big Five Aspect Scales (BFAS). (Manuscript in Preparation).

Nishiguchi, Y., Takano, K., \& Tanno, Y. (2015). Explicitly guided attentional bias modification promotes attentional disengagement from negative stimuli. Emotion, 15, 731-741. https://doi.org/10.1037/a0039117.

Osinsky, R., Wilisz, D., Kim, Y., Karl, C., \& Hewig, J. (2014). Does a single session of attentional bias modification influence early neural mechanisms of spatial attention? An ERP study. Psychophysiology, 51, 982-989. https://doi.org/10.1111/psyp.12247.

Ossandón, J. P., Onat, S., \& König, P. (2014). Spatial biases in viewing behavior. Journal of Vision, 14https://doi.org/10.1167/14.2.20.

Peirce, J. W. (2007). PsychoPy—psychophysics software in Python. Journal of Neuroscience Methods, 162, 8-13.

Price, R. B., Greven, I. M., Siegle, G. J., Koster, E. H. W., \& Raedt, R. de (2015). A novel attention training paradigm based on operant conditioning of eye gaze: Preliminary findings. Emotion (Washington, D.C.). Advance online publicationhttps://doi.org/10. $1037 /$ emo0000093.

Price, R. B., Wallace, M., Kuckertz, J. M., Amir, N., Graur, S., Cummings, L., ... Bar-Haim, Y. (2016). Pooled patient-level meta-analysis of children and adults completing a computer-based anxiety intervention targeting attentional bias. Clinical Psychology Review, 50, 37-49. https://doi.org/10.1016/j.cpr.2016.09.009.

Prime, D. J., Pluchino, P., Eimer, M., Dell'Acqua, R., \& Jolicœur, P. (2011). Object-substitution masking modulates spatial attention deployment and the encoding of information in visual short-term memory: Insights from occipito-parietal ERP components. Psychophysiology, 48, 687-696. https://doi.org/10.1111/j.1469-8986.2010. 01133.x.

Puls, S., \& Rothermund, K. (2017). Attending to emotional expressions: No evidence for automatic capture in the dot-probe task. Cognition \& Emotion, 1-14. https://doi.org/ 10.1080/02699931.2017.1314932.

Reutter, M., Hewig, J., Wieser, M. J., \& Osinsky, R. (2017). The N2pc component reliably captures attentional bias in social anxiety. Psychophysiology, 54, 519-527. https:// doi.org/10.1111/psyp.12809.

Robitaille, N., \& Jolicœur, P. (2006). Fundamental properties of the N2pc as an index of spatial attention: Effects of masking. Canadian Journal of Experimental Psychology/ Revue Canadienne De Psychologie Expérimentale, 60, 101-111. https://doi.org/10. $1037 /$ cjep2006011.

Rodebaugh, T. L., Scullin, R. B., Langer, J. K., Dixon, D. J., Huppert, J. D., Bernstein, A., ... Lenze, E. J. (2016). Unreliability as a threat to understanding psychopathology: The cautionary tale of attentional bias. Journal of Abnormal Psychology, 125, 840-851. https://doi.org/10.1037/abn0000184.

Rossignol, M., Campanella, S., Bissot, C., \& Philippot, P. (2013). Fear of negative evaluation and attentional bias for facial expressions: An event-related study. Brain and Cognition, 82, 344-352. https://doi.org/10.1016/j.bandc.2013.05.008.

Rossion, B., Joyce, C. A., Cottrell, G. W., \& Tarr, M. J. (2003). Early lateralization and orientation tuning for face, word, and object processing in the visual cortex. NeuroImage, 20, 1609-1624. https://doi.org/10.1016/j.neuroimage.2003.07.010.

Schmidt, N. B., Richey, J. A., Buckner, J. D., \& Timpano, K. R. (2009). Attention training for generalized social anxiety disorder. Journal of Abnormal Psychology, 118, 5-14. https://doi.org/10.1037/a0013643.

Schmitt, M., \& Maes, J. (2000). Vorschlag zur Vereinfachung des Beck-DepressionsInventars (BDI). Diagnostica, 46, 38-46. https://doi.org/10.1026//0012-1924.46. 1.38 .

Schmukle, S. C. (2005). Unreliability of the dot probe task. European Journal of Personality, 19, 595-605. https://doi.org/10.1002/per.554.

Schneider, D., Hoffmann, S., \& Wascher, E. (2014). Sustained posterior contralateral activity indicates re-entrant target processing in visual change detection: An EEG study. Frontiers in Human Neuroscience, 8, 247. https://doi.org/10.3389/fnhum.2014. 00247.

See, J., MacLeod, C., \& Bridle, R. (2009). The reduction of anxiety vulnerability through the modification of attentional bias: A real-world study using a home-based cognitive bias modification procedure. Journal of Abnormal Psychology, 118, 65-75. https://doi org/10.1037/a0014377.

Shechner, T., \& Bar-Haim, Y. (2016). Threat monitoring and attention-bias modification in anxiety and stress-related disorders. Current Directions in Psychological Science, 25, 431-437. https://doi.org/10.1177/0963721416664341.

Sigurjónsdóttir, Ó., Björnsson, A. S., Ludvigsdóttir, S. J., \& Kristjánsson, Á. (2015). Money talks in attention bias modification: Reward in a dot-probe task affects attentional 
biases. Visual Cognition, 23, 118-132. https://doi.org/10.1080/13506285.2014. 984797.

Spielberger, C. D. (2010). State-Trait anxiety inventory. Wiley Online Library.

Stangier, U., Heidenreich, T., Berardi, A., Golbs, U., \& Hoyer, J. (1999). Assessment of social phobia by the social interaction anxiety scale and the social phobia scale. Zeitschrift Fur Klinische Psychologie-Forschung Und Praxis, 28, 28-36.

Staugaard, S. R. (2009). Reliability of two versions of the dot-probe task using photographic faces. Psychology Science Quarterly, 51, 339-350.

Taylor, C. T., Aupperle, R. L., Flagan, T., Simmons, A. N., Amir, N., Stein, M. B., et al. (2014). Neural correlates of a computerized attention modification program in anxious subjects. Social Cognitive and Affective Neuroscience, 9, 1379-1387. https://doi. org/10.1093/scan/nst128.

Toga, A. W., \& Thompson, P. M. (2003). Mapping brain asymmetry. Nature Reviews Neuroscience, 4, 37-48. https://doi.org/10.1038/nrn1009.

Torrence, R. D., Wylie, E., \& Carlson, J. M. (2017). The time-course for the capture and hold of visuospatial attention by fearful and happy faces. Journal of Nonverbal Behavior, 41, 139-153. https://doi.org/10.1007/s10919-016-0247-7.

Turner, S. M., Beidel, D. C., Dancu, C. V., \& Stanley, M. A. (1989). An empirically derived inventory to measure social fears and anxiety: The social phobia and anxiety inventory. Psychological Assessment: A Journal of Consulting and Clinical Psychology, 1,35 .

Van Bockstaele, B., Verschuere, B., Koster, E. H. W., Tibboel, H., Houwer, J. de, \& Crombez, G. (2011). Differential predictive power of self report and implicit measures on behavioural and physiological fear responses to spiders. International Journal of Psychophysiology : Official Journal of the International Organization of Psychophysiology, 79, 166-174. https://doi.org/10.1016/j.ijpsycho.2010.10.003.

Waechter, S., Nelson, A. L., Wright, C., Hyatt, A., \& Oakman, J. (2014). Measuring attentional bias to threat: Reliability of dot probe and eye movement indices. Cognitive
Therapy and Research, 38, 313-333. https://doi.org/10.1007/s10608-013-9588-2.

Waechter, S., \& Stolz, J. A. (2015). Trait anxiety, state anxiety, and attentional bias to threat: Assessing the psychometric properties of response time measures. Cognitive Therapy and Research, 39, 441-458. https://doi.org/10.1007/s10608-015-9670-z.

Wascher, E., \& Beste, C. (2010). Tuning perceptual competition. Journal of Neurophysiology, 103, 1057-1065. https://doi.org/10.1152/jn.00376.2009.

Weymar, M., Gerdes, A. B. M., Löw, A., Alpers, G. W., \& Hamm, A. O. (2013). Specific fear modulates attentional selectivity during visual search: Electrophysiological insights from the N2pc. Psychophysiology, 50, 139-148. https://doi.org/10.1111/psyp.12008.

Weymar, M., Löw, A., Ohman, A., \& Hamm, A. O. (2011). The face is more than its partsbrain dynamics of enhanced spatial attention to schematic threat. NeuroImage, 58, 946-954. https://doi.org/10.1016/j.neuroimage.2011.06.061.

White, L. K., Britton, J. C., Sequeira, S., Ronkin, E. G., Chen, G., Bar-Haim, Y., ... Pine, D. S. (2016). Behavioral and neural stability of attention bias to threat in healthy adolescents. NeuroImage, 136, 84-93. https://doi.org/10.1016/j.neuroimage.2016.04. 058.

Wieser, M. J., Hambach, A., \& Weymar, M. (2018). Neurophysiological correlates of attentional bias for emotional faces in socially anxious individuals - evidence from a visual search task and N2pc. Biological Psychology, 132, 192-201. https://doi.org/10. 1016/j.biopsycho.2018.01.004.

Wieser, M. J., \& Moscovitch, D. A. (2015). The effect of affective context on visuocortical processing of neutral faces in social anxiety. Frontiers in Psychology, 6, 1824. https:// doi.org/10.3389/fpsyg.2015.01824.

Wittchen, H. U., Jacobi, F., Rehm, J., Gustavsson, A., Svensson, M., Jönsson, B., ... Steinhausen, H.-C. (2011). The size and burden of mental disorders and other disorders of the brain in Europe 2010. European Neuropsychopharmacology : The Journal of the European College of Neuropsychopharmacology, 21, 655-679. https://doi.org/10. 1016/j.euroneuro.2011.07.018. 